\title{
Ethno-botanical Survey on Medicinal plants used by Tribes of Karanja (Ghadge) Tahsil of Wardha District, Maharashtra, India
}

\author{
Research Article
}

\section{Bharat Rathi1 $^{*}$, Pramod Khobragade ${ }^{2}$, Renu Rathi ${ }^{3}$, Rajkumar Gupta ${ }^{4}$}

1. Professor, Department of Rasashastra \& Bhaishajya Kalpana, 2. Professor \& HOD, Department of Dravyaguna, 3. Professor, Department of Kaumarbhritya, 4. Professor, Department of Dravyaguna, Mahatma Gandhi Ayurveda College Hospital \& Research Centre, Salod (H) Wardha (MS) - 442001.

\begin{abstract}
Introduction: Tribals or forest dwellers have much knowledge of the drugs growing around them. Most of this treasure of knowledge has been passed orally without any written documents. It is therefore very important to preserve and protect the traditional knowledge and also to prepare a digital data base of traditional medicine. Aim \& Objectives: Ethno-botanical survey on medicinal Plants used by the Tribes of Karanja (Ghadge) Tahsil of Wardha District, Maharashtra, India and to prepare the ethno-botanical database of study area. Material \& Methods: Ethnobotanical study was conducted through field surveys. Data was collected using questionnaire, interviews and discussions. The collected data was investigated using specific quantitative parameters including Informant Consensus Factor (ICF), Relative popularity level (RPL), Use value (UV), Fidelity level (FL), Relative frequency citation (RFC) and Rank order priority (ROP). Observations \& results: Total 7 local informants were interviewed for Ethno-botanical data. Total 65 plants associated to 46 families were verified, authenticated and then recognized with ethno medicinal uses. Informants used 10 types of parts of plant from 6 kind of habitat and do the management of the patients with 6 type of medicinal preparations through 5 routes of administrations. Values obtained by calculating ICF, UV, RFC, FL, RPL and ROP were suggestive of informant's knowledge regarding particular plant species in treating the particular ailment. Conclusion: The present work would be useful in identification of newer species and their therapeutic applications.
\end{abstract}

Key Words: Ethnobotany, Ethnomedicine, Informants, Survey, Plant species.

\section{Introduction}

The importance of Aushadhi (drug) and its use as a medicine is well known since Vedic period. Indian systems of medicine are part of our cultural heritage and have rendered cost effective and efficient patient management through the ages. The very first description of medicine can be found in Rigveda which is one of the oldest literatures that describes the significance of 67 medicinal plants (1). Charaka Samhita and Sushruta Samhita holds commendable knowledge on therapeutic applications of near about 500 medicinal plants described in various chapters. Acharya Charaka has explained the significance of herbal plants in Sutrasthana $26^{\text {th }}$ chapter. Acharya Charaka has opinion that in this world there is not a single herb which does not have therapeutic value, unless it is used in apt conditions and after proper processing. Charaka also mentioned about the habitat of medicinal plants (2).

\section{* Corresponding Author:}

\section{Bharat Rathi}

Professor, Dept of Rasashastra \& Bhaishajya Kalpana, Mahatma Gandhi Ayurveda College Hospital \& Research Centre, Salod (H),

Wardha (MS) - 442001. India.

Email Id: bharatrathi174@gmail.com
It is well known that the forest dwellers including the cattle grazers etc. have had a sound known knowledge of the morphology and properties of the drugs growing around them (3). Tribals even now have much knowledge of the drugs, a knowledge which they have inherited traditionally through ages. This fact has also been fully supported by the following reference in Atharvaveda, where it has been mentioned that the Kirata (one of the tribals) used to collect drugs.

Acharya Sushruta also mentioned the source of knowledge about medicinal plants. To his opinion, curative herbs and plants should be identified and approved with the help of cattle grazers, Goatherds, shepherd, hunters, forest-dwellers who roam in forest and collect the useful parts such as fruits, roots, stem etc. of plants (4). Thus traditional healers have been the major source of protecting rich heritage of understanding on medicinal plants and their therapeutic usage. They have both the know-how and do-how for preparation and administration of medicine. In India, it is estimated that traditional healers use 2500 plant species grow around them as regular source of medicine to treat various disease conditions (5-6). Most of this treasure of knowledge is transferred to next generation orally without any written documents and is still retained by them (7). Thus people throughout the globe use medicinal herbal plants as a medicine for treating the majority of their ailments. Increase in side effects with modern drugs, high cost for treating the general 
Bharat Rathi et.al., Ethno-botanical Survey on Medicinal plants used by Tribes of Karanja (Ghadge) Tahsil

diseases, increasing population are some of the reasons for growing interest in the use of herbs as source of medicine (8). Plants, animal and mineral products are major source for the basic conventional knowledge of Ayurvedic medicine. Ethno-botanical studies have been augmented in recent times at the regional, national and international platform. Literature searches reveal that there is still a massive gap in awareness of ethnomedicine and its scientific authentication. It is therefore very essential to preserve and protect the traditional knowledge and also to establish a digital data base of traditional medicine. If this information is yet to be collected systematically and comprehensively and maintained in databases would be a big contribution. Considering this fact, present work was undertaken to explore and prepare the ethno botanical and ethno medicinal database on medicinal plants used by the tribal people of Karanja (Ghadge) tahsil in Wardha district of Maharashtra, India. Most of the tribal population resided in various villages of this tahsil and prefer traditional healers for their treatment.

\section{Material \& Methods \\ Study area}

Karanja (Ghadge) town is the head quarter of Karanja tahsil which is located $59 \mathrm{Km}$ towards North from District head quarter Wardha, $76 \mathrm{~km}$ away from Nagpur and $79 \mathrm{~km}$ away from Amravati. Karanja Taluka is bounded by by Arvi Taluka towards South, Ashti Taluka towards west, Katol Taluka towards East, Narkhed Taluka towards North. Karanja consist of 101 Villages and 60 Panchayats. Karanja is located at coordinates $21^{\circ} 10^{\prime} 58.38^{\prime \prime}$ North Latitude and $78^{\circ} 22^{\prime} 52.75$ East Longitude and 415 meters above sea level. It is too hot in summer the highest day temperature is in between $31^{\circ} \mathrm{C}$ to $47.5^{\circ} \mathrm{C}$. Most of the regions in Karanja tahsil areas are covered with Semi evergreen forest inhabited by the rural population of tribal people. In this forest region number of herbal plants rich with medicinal properties are found which are used by the tribal people for curing various ailments.

\section{Sampling Informants}

In Karanja Ghadge tahsil tribal communities dwell in the villages like Belara tanda, Borgaon godi, Brahmanwada, Dhamkund, Dhanoli, Garpit, Hetikundi, Hararasi, Hiwara Tanda, Khairwada, Ladgad,Linga, Methiwari, Manjara, Masod and Chopan. Most of the tribes have thorough information of medicinal plants and collect plants from adjacent forest to cure their ailments and diseases.
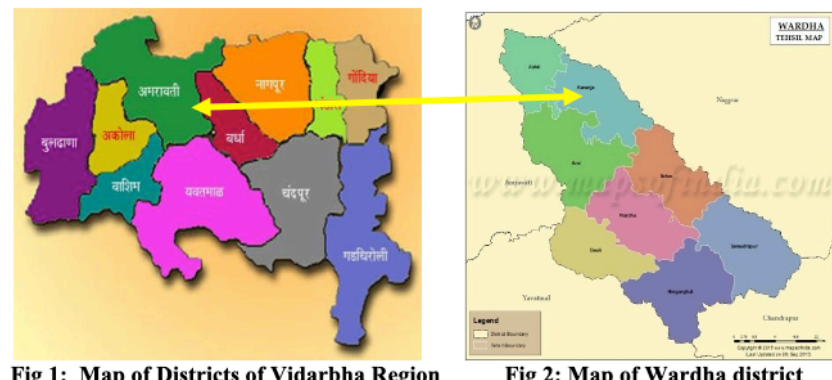

Fig 2: Map of Wardha district

\section{Ethnobotanical Data collection}

Ethno-botanical study was conducted through field surveys during September 2018- April 2019 in different remote villages of the Karanja (Ghadge) tahsil in Wardha district where the tribal communities lived with their customs and rituals. The purpose of the study was obviously explained and verbal consent was taken from each informant.

Data was collected using questionnaire, personal interviews and discussions in their local language. Samples of traditional medicines were collected from the tribal and experienced individuals practicing indigenous medicines. Meeting with tribal were arranged by local people, forest department personnel who were familiar and could communicate with them in their own language. The questionnaires used included the following information: Common name, habit, useful parts of plant, type of preparation, mode of application, dose and their medicinal uses. The actual field photographs of the wild plants with their surroundings were taken. The herbal drugs utilized by the traditional healers were authenticated with the help of taxonomist, forest range officer and database on medicinal plants used in Ayurveda. Their specific medicinal values were verified with the help of literature review. Few specimens were collected and prepared according to standard herbarium procedures. Data was analyzed by descriptive statistics.

\section{Quantitative analysis of ethno botanical data}

The collected data was investigated using specific quantitative parameters including Informant Consensus Factor (ICF), Relative popularity level (RPL), Use value (UV), Fidelity level (FL), Relative frequency citation (RFC) and Rank order priority (ROP). Obtained values are depicted in numbers and percentages.

\section{Informant consensus factor (ICF)}

It was developed by Trotter and Logan which tests the consistency of informant's knowledge regarding plants species for treating a particular illness category. This parameter accounts for the degree of agreement among the different informants interviewed concerning the use.

The ICF value is calculated by using the formula (9).

$$
\mathrm{ICF}=\frac{\mathrm{N} u r-\mathrm{N} t}{\mathrm{~N} u r-1}
$$

Where, Nur is the total number of use reports for each disease category and $\mathrm{N} t$ indicates the number of species used in said category.

Use value (UV): Use Value counts the importance of each plant on the basis of the number of different uses reported. Use value gives an idea about the important species used by a community. It is calculated using the formula (10).

$$
\mathrm{UV} i=\frac{\sum \mathrm{U} i}{\mathrm{~N}}
$$


Where, UV indicates use value of individual species, $\sum \mathrm{U} i$ is the sum of the uses recoded for

that species and $\mathrm{N}$ represents the number of informants who reported that species.

\section{Relative frequency of citation (RFC)}

Relative frequency of citation (RFC) signifies the local importance of each species in a study area. The most popularly used plant species will get the highest number for the citation/frequency among the community members. This index is determined by dividing the number of informants citing a useful species (FC) by total number of informants in the survey $(\mathrm{N})$. RFC is calculated by the formula (11).

$$
\mathrm{RFC}=\frac{\mathrm{FC}}{\mathrm{N}} 0<R F C<1 .
$$

Theoretically, it varies from zero to 1 . When few informants quote the species a value close to zero is obtained. The upper limit one is seldom obtained, it is possible only when all the informants quote a particular species.

\section{Fidelity level (FL)}

FL is the percentage of informants who claim the uses of certain plant species to treat a particular ailment in a study area. The FL index is calculated using formula (12).

$$
\text { FL }(\%)=\frac{\mathrm{Np}}{\mathrm{N}}
$$

Where, ' $\mathrm{Np}$ ' is the number of informants who claimed a use of certain plant species for a particular disease and ' $\mathrm{N}$ ' is the total number of informants citing the species for any given disease. The maximum FL indicates the frequency and high use of the plant species for treating a particular ailment by the informants of the study area.

\section{Relative popularity level (RPL)}

RPL is the ratio between numbers of ailments treated by a particular plant species and the total number of informants for any disease. The relative popularity level (RPL) assumes a value 0 and 1.0, with ' 1 ' being complete popularity of a plant for major ailments and ' 0 ' no ailments treated by a plant species (13).

\section{Rank order priority (ROP)}

ROP is a correction factor, used for appropriate ranking of the plant species with different FL and RPL values. The ROP is derived by multiplying RPL and FL values as explained earlier (14).

$$
\mathrm{ROP}=\mathrm{FL} \text { RPL }
$$

\begin{tabular}{|c|c|c|c|c|c|c|c|c|c|c|c|}
\hline S.N. & $\begin{array}{l}\text { Botanical } \\
\text { name }\end{array}$ & Family & $\begin{array}{l}\text { Local } \\
\text { Name }\end{array}$ & $\begin{array}{l}\text { Sanskrit } \\
\text { name }\end{array}$ & Habit & $\begin{array}{l}\text { Part } \\
\text { Used }\end{array}$ & $\begin{array}{l}\text { Disease } \\
\text { Treated }\end{array}$ & $\begin{array}{l}\text { Preparatio } \\
\text { n }\end{array}$ & $\begin{array}{l}\text { Administ } \\
\text { ration } \\
\text { Route }\end{array}$ & RFC* & $\mathbf{U V} * *$ \\
\hline 1 & $\begin{array}{l}\text { Acacia arabica } \\
\text { (Lam.)Willd. }\end{array}$ & Mimosoideae & Babhul & Babbul & Tree & $\begin{array}{l}\text { Bark, } \\
\text { Gum }\end{array}$ & $\begin{array}{l}\text { Pyorrhea, } \\
\text { General } \\
\text { debility }\end{array}$ & $\begin{array}{l}\text { Decoction, } \\
\text { Powder }\end{array}$ & $\begin{array}{l}\text { Oral, } \\
\text { Gargle }\end{array}$ & 0.14 & 0.28 \\
\hline 2 & $\begin{array}{l}\text { Achyranthes } \\
\text { aspera Linn. }\end{array}$ & Amaranthaceae & Aghada & Apamarga & Herb & Root & $\begin{array}{l}\text { Dogbite, } \\
\text { Snake bite, } \\
\text { Scorpion bite, }\end{array}$ & $\begin{array}{l}\text { Decoction, } \\
\text { Paste }\end{array}$ & Topical & 0.28 & 0.57 \\
\hline 3 & $\begin{array}{l}\text { Adhatoda } \\
\text { vasica } \text { Nees }\end{array}$ & Acanthaceae & Adusa & Vasa & Shrub & Leaves & $\begin{array}{l}\text { Asthama, } \\
\text { Phelgm }\end{array}$ & $\begin{array}{l}\text { Juice with } \\
\text { honey }\end{array}$ & Oral & 0.57 & 0.85 \\
\hline 4 & $\begin{array}{l}\text { Aegel } \\
\text { marmelos } \\
\text { Linn. }\end{array}$ & Rutaceae & $\mathrm{Bel}$ & Bilwa & Tree & Fruit & $\begin{array}{l}\text { Diarrhoea, } \\
\text { amaebiaasis }\end{array}$ & Powder & Oral & 0.14 & 0.28 \\
\hline 5 & $\begin{array}{l}\text { Ailanthus } \\
\text { excelsa } \mathrm{Roxb}\end{array}$ & Simaroubaceae & Maharukh & Aralu & Tree & $\begin{array}{l}\text { Bark, } \\
\text { Leaves }\end{array}$ & $\begin{array}{l}\text { Asthama, } \\
\text { Cr.Fever, } \\
\text { Tonic }\end{array}$ & Powder & Oral & 0.28 & 0.85 \\
\hline 6 & $\begin{array}{l}\text { Aloe vera (L.) } \\
\text { Burn.F. }\end{array}$ & Asphodelaceae & $\begin{array}{l}\text { Karan } \\
\text { kuwari }\end{array}$ & Kumari & Herb & Leaves & $\begin{array}{l}\text { Wound, } \\
\text { burns, } \\
\text { amenorrhoea }\end{array}$ & Paste & $\begin{array}{l}\text { Oral, } \\
\text { Topical }\end{array}$ & 0.14 & 0.42 \\
\hline 7 & $\begin{array}{l}\text { Allium } \\
\text { ursenum L. }\end{array}$ & Amaryllidaceae & $\begin{array}{l}\text { Jangali } \\
\text { Lashun }\end{array}$ & $\begin{array}{l}\text { Wild } \\
\text { Lashun }\end{array}$ & Herb & Tuber & Galectogauge & Decoction & Oral & 0.14 & 0.14 \\
\hline 8 & $\begin{array}{l}\text { Anogeissus } \\
\text { latifolia(DC)W } \\
\text { all }\end{array}$ & Combretaceae & Dhavada & Dhav & Tree & Bark & $\begin{array}{l}\text { Intestinal } \\
\text { ulcer }\end{array}$ & Decoction & Oral & 0.14 & 0.14 \\
\hline 9 & $\begin{array}{l}\text { Andrographis } \\
\text { paniculata } \\
\text { (Burm.F.)Nees }\end{array}$ & Acanthaceae & Bhuineem & $\begin{array}{l}\text { Kalmegh, } \\
\text { Chiraita }\end{array}$ & Herb & $\begin{array}{l}\text { Whole } \\
\text { plant }\end{array}$ & Fever, Acidity & Decoction & Oral & 0.14 & 0.28 \\
\hline 10 & $\begin{array}{l}\text { Argemone } \\
\text { mexicana Linn }\end{array}$ & Papaveraceae & $\begin{array}{l}\text { Pivala } \\
\text { dhotra }\end{array}$ & $\begin{array}{l}\text { Swarna } \\
\text { kshiri }\end{array}$ & Shrub & $\begin{array}{l}\text { Root, } \\
\text { Leaves }\end{array}$ & Skin diseases & Ash & Topical & 0.14 & 0.14 \\
\hline 11 & $\begin{array}{l}\text { Argyreia } \\
\text { speciosa Linn } \\
\text { F. }\end{array}$ & Convolvulaceae & Vidhara & $\begin{array}{l}\text { Samudr } \\
\text { shosha }\end{array}$ & Climber & Seeds & $\begin{array}{l}\text { Aphrodisiac, } \\
\text { Joint pain }\end{array}$ & Powder & Oral & 0.14 & 0.28 \\
\hline 12 & $\begin{array}{l}\text { Arundo } \\
\operatorname{donax}(\mathrm{L})\end{array}$ & Gramineae & $\begin{array}{l}\text { Palicha } \\
\text { kanda, } \\
\text { San }\end{array}$ & Ranboru & Herb & Tuber & Piles, Eczema & Paste & Topical & 0.28 & 0.42 \\
\hline 13 & $\begin{array}{l}\text { Asparagus } \\
\text { recemosus } \\
\text { (willd) }\end{array}$ & Asparagaceae & Sansan & Shatavari & Climber & Root & $\begin{array}{l}\text { Antacid, } \\
\text { Lactation, } \\
\text { Tonic }\end{array}$ & Powder & Oral & 0.14 & 0.42 \\
\hline
\end{tabular}

\section{Observation \& Results}

Table 1: List of Ethno-botanical plant species of the study area with RFC and UV value: 
Bharat Rathi et.al., Ethno-botanical Survey on Medicinal plants used by Tribes of Karanja (Ghadge) Tahsil

\begin{tabular}{|c|c|c|c|c|c|c|c|c|c|c|c|}
\hline 14 & $\begin{array}{l}\text { Astercantha } \\
\text { longifolia (L.) } \\
\text { Ness }\end{array}$ & Acanthaceae & Kolistha & $\begin{array}{l}\text { Talim } \\
\text { khana } \\
\text { Kokil- } \\
\text { aksha }\end{array}$ & Herb & Seeds & $\begin{array}{l}\text { Aphrodisiac, } \\
\text { Tonic }\end{array}$ & Powder & Oral & 0.14 & 0.28 \\
\hline 15 & $\begin{array}{l}\text { Azadirachta } \\
\text { indica A.Juss }\end{array}$ & Maliaceae & Neem & $\mathrm{Nimb}$ & Tree & Bark & Fever & Decoction & Oral & 0.14 & 0.14 \\
\hline 16 & $\begin{array}{l}\text { Bacopa } \\
\text { monnieri } \\
\text { H.B.K. }\end{array}$ & Plantaginaceae & $\begin{array}{l}\text { Dimakh- } \\
\text { dari }\end{array}$ & Bramhi & Herb & Plant & $\begin{array}{l}\text { Memory } \\
\text { enhancer, } \\
\text { Nervous } \\
\text { disorder }\end{array}$ & Paste & Topical & 0.28 & 0.71 \\
\hline 17 & $\begin{array}{l}\text { Balanitis } \\
\text { aegyptica(L.) } \\
\text { Delil }\end{array}$ & Zygophyllaceae & Hinganbet & Ingudi & Tree & Fruit & Worms & Decoction & Oral & 0.14 & 0.14 \\
\hline 18 & $\begin{array}{l}\text { Bauhinia } \\
\text { recemosa Lam. }\end{array}$ & Leguminoceae & Bhos & $\begin{array}{l}\text { Ashma- } \\
\text { ntak }\end{array}$ & Tree & Leaves & $\begin{array}{l}\text { Burning } \\
\text { micturation, } \\
\text { Renal calculi }\end{array}$ & Juice & Oral & 0.14 & 0.28 \\
\hline 19 & $\begin{array}{l}\text { Brassica } \\
\text { juncea (L.) } \\
\text { Czern }\end{array}$ & Cruciferae & Aasuri & Rajika & Herb & Flower & $\begin{array}{l}\text { Asthama, } \\
\text { Phelgm }\end{array}$ & $\begin{array}{l}\text { Ash with } \\
\text { honey }\end{array}$ & Oral & 0.14 & 0.28 \\
\hline 20 & $\begin{array}{l}\text { Butea } \\
\text { monosperma } \\
\text { Lam. }\end{array}$ & Papilionaceae & Palasful & Palash & Tree & Flower & Diabetes & Decoction & Oral & 0.14 & 0.14 \\
\hline 21 & $\begin{array}{l}\text { Cassia } \\
\text { angustifolia } \\
\text { Vahl }\end{array}$ & Leguminoceae & $\begin{array}{l}\text { Sonaa- } \\
\text { mukhi }\end{array}$ & $\begin{array}{l}\text { Marka- } \\
\text { ndika }\end{array}$ & Climber & Leaves & Constipation & Powder & Oral & 0.14 & 0.14 \\
\hline 22 & Cassia tora $\mathrm{L}$. & Leguminoceae & $\begin{array}{l}\text { Tarota, } \\
\text { Takla }\end{array}$ & $\begin{array}{l}\text { Chakra } \\
\text { marda }\end{array}$ & Herb & Seeds & $\begin{array}{l}\text { Abdominal } \\
\text { pain, Itching }\end{array}$ & Decoction & $\begin{array}{l}\text { Oral, } \\
\text { Tropical }\end{array}$ & 0.14 & 0.28 \\
\hline 23 & $\begin{array}{l}\text { Calotropis } \\
\text { gigantea (L.) }\end{array}$ & Asclepideaceae & $R u i$ & Arka & Shrub & $\begin{array}{l}\text { Flower } \\
\text { s }\end{array}$ & $\begin{array}{l}\text { Asthama, } \\
\text { Pheglm }\end{array}$ & Powder & Oral & 0.14 & 0.28 \\
\hline 24 & $\begin{array}{l}\text { Capparis } \\
\text { zeylanica }(\mathrm{L} .)\end{array}$ & Capparaceae & Vaghanti & $\begin{array}{l}\text { Vyaghra } \\
\text { nakhi }\end{array}$ & Herb & Root & Dog bite & Paste & Topical & 0.14 & 0.14 \\
\hline 25 & $\begin{array}{l}\text { Celastrus } \\
\text { paniculatus } \\
\text { (Willd.) }\end{array}$ & Celastraceae & Fofus vel & $\begin{array}{l}\text { Jyotish- } \\
\text { mati }\end{array}$ & Climber & $\begin{array}{l}\text { Seeds, } \\
\text { Tuber }\end{array}$ & $\begin{array}{l}\text { Aphrodisiac } \\
\text { Tonic }\end{array}$ & Powder & Oral & 0.14 & 0.28 \\
\hline 26 & $\begin{array}{l}\text { Citrulus } \\
\text { colocynthis } \\
\text { (L.) Schrad }\end{array}$ & Cucurbitaceae & $\begin{array}{l}\text { Jangali } \\
\text { sherni, } \\
\text { safed } \\
\text { indryan }\end{array}$ & $\begin{array}{l}\text { Indra- } \\
\text { waruni }\end{array}$ & Creeper & $\begin{array}{l}\text { Seeds, } \\
\text { Root }\end{array}$ & $\begin{array}{l}\text { Worms, } \\
\text { Dismehhoragi } \\
\text { a } \\
\text { Snake bite }\end{array}$ & Powder & Oral & 0.28 & 0.71 \\
\hline 27 & $\begin{array}{l}\text { Cletoria } \\
\text { ternaeta }(\mathrm{L} .)\end{array}$ & Fabaceae & $\begin{array}{l}\text { Safed } \\
\text { Bhopari }\end{array}$ & Aparajita & Climber & $\begin{array}{l}\text { Whole } \\
\text { plant }\end{array}$ & Azoospermia & Powder & Oral & 0.14 & 0.14 \\
\hline 28 & $\begin{array}{l}\text { Cochlospermu } \\
m \text { religiosum } \\
\text { (L.) } \\
\text { Alston }\end{array}$ & Bixaceae & Gongal & $\begin{array}{l}\text { Giri- } \\
\text { salmalika } \\
\text { Ganeri }\end{array}$ & Tree & Gum & $\begin{array}{l}\text { Cough, } \\
\text { Diarrhoea, } \\
\text { Dysentry }\end{array}$ & Powder & Oral & 0.14 & 0.42 \\
\hline 29 & $\begin{array}{l}\text { Crateava } \\
\text { nurvala Buch. } \\
\text { Hum }\end{array}$ & Capparidaceae & $\begin{array}{l}\text { Varna } \\
\text { varni }\end{array}$ & Vayvarna & Tree & $\begin{array}{l}\text { Bark, } \\
\text { Leaves }\end{array}$ & $\begin{array}{l}\text { Internal } \\
\text { abscess }\end{array}$ & $\begin{array}{l}\text { Decoction, } \\
\text { Paste }\end{array}$ & $\begin{array}{l}\text { Oral, } \\
\text { external }\end{array}$ & 0.14 & 0.14 \\
\hline 30 & $\begin{array}{l}\text { Curcuma } \\
\text { amada } \\
\text { Roxb }\end{array}$ & Zingiberaceae & $\begin{array}{l}\text { Ambehala } \\
d\end{array}$ & $\begin{array}{l}\text { Amra- } \\
\text { haridra }\end{array}$ & Herb & Tuber & $\begin{array}{l}\text { Diseases of } \\
\text { nervous } \\
\text { system, } \\
\text { Itching }\end{array}$ & Paste & Tropical & 0.14 & 0.28 \\
\hline 31 & $\begin{array}{l}\text { Cuscuta } \\
\text { reflexa (Roxb) }\end{array}$ & Convolvulaceae & Adharvel & Aakashvel & Climber & $\begin{array}{l}\text { Whole } \\
\text { plant }\end{array}$ & Skin diseases & Ash & Topical & 0.14 & 0.14 \\
\hline 32 & $\begin{array}{l}\text { Cymbopogon } \\
\text { martini Roxb }\end{array}$ & Gramineae & $\begin{array}{l}\text { Tikhadaza } \\
\text { ra }\end{array}$ & Tikhadi & Grass & Leaves & Fever & Decoction & Oral & 0.14 & 0.14 \\
\hline 33 & $\begin{array}{l}\text { Cynodon } \\
\text { dactylon }(\mathrm{L}) . \\
\text { Pers }\end{array}$ & Poaceae & Doob & Durva & Grass & Leaves & $\begin{array}{l}\text { Epistaxis, } \\
\text { Fever }\end{array}$ & Juice & $\begin{array}{l}\text { Nasal } \\
\text { drop, } \\
\text { oral }\end{array}$ & 0.14 & 0.28 \\
\hline 34 & $\begin{array}{l}\text { Dioscorea } \\
\text { bulbifera( L.) }\end{array}$ & Discoreaceae & $\begin{array}{l}\text { Dukkarka } \\
\text { nda }\end{array}$ & Warahi & Creeper & Tuber & $\begin{array}{l}\text { All skin } \\
\text { diseases }\end{array}$ & Paste & Topical & 0.14 & 0.14 \\
\hline 35 & $\begin{array}{l}\text { Diplocyclos } \\
\text { palmatus } \mathrm{L} .\end{array}$ & Cucurbitaceae & Shivlingi & Shivlingi & Climber & Seeds & $\begin{array}{l}\text { Female } \\
\text { infertility }\end{array}$ & Powder & Oral & 0.14 & 0.14 \\
\hline 36 & $\begin{array}{l}\text { Dregea } \\
\text { volubilis (L.f) }\end{array}$ & Asclepiadaceae & Hiranvel & $\begin{array}{l}\text { Haran- } \\
\text { dodi }\end{array}$ & Creeper & Root & $\begin{array}{l}\text { Lactation, } \\
\text { aphrodisiac }\end{array}$ & Powder & Oral & 0.14 & 0.28 \\
\hline 37 & $\begin{array}{l}\text { Ehretia Laevis } \\
\text { Roxb.) }\end{array}$ & Boraginaceae & $\begin{array}{l}\text { Khanduch } \\
\text { akka }\end{array}$ & $\begin{array}{l}\text { Khandu- } \\
\text { chakka }\end{array}$ & Tree & Leaves & Wound & Powder & Topical & 0.14 & 0.14 \\
\hline 38 & $\begin{array}{l}\text { Euforbia } \\
\text { nerifolia (L.) }\end{array}$ & Euphorbiceae & Nagphani & Snuhi & Shrub & Leaves & $\begin{array}{l}\text { Itching, } \\
\text { Eczema }\end{array}$ & $\begin{array}{l}\text { Ash with } \\
\text { coconut oil }\end{array}$ & Tropical & 0.28 & 0.42 \\
\hline 39 & $\begin{array}{l}\text { Eugenia } \\
\text { jambolana } \\
\text { Lam. }\end{array}$ & Myrtaceae & Jambhul & Jamuna & Tree & $\begin{array}{l}\text { Seeds, } \\
\text { Leaves }\end{array}$ & $\begin{array}{l}\text { Diabetis, } \\
\text { Diarrhoea }\end{array}$ & $\begin{array}{l}\text { Powder } \\
\text { Juice }\end{array}$ & Oral & 0.14 & 0.28 \\
\hline 40 & $\begin{array}{l}\text { Gmelina } \\
\text { arborea Roxb }\end{array}$ & Laminaceae & Shivan & Gambhari & Tree & Leaves & $\begin{array}{l}\text { Meningitis, } \\
\text { Cr. rhinitis, } \\
\text { Epilepsy }\end{array}$ & $\begin{array}{l}\text { Paste, } \\
\text { Juice }\end{array}$ & $\begin{array}{l}\text { Topical, } \\
\text { Nasal } \\
\text { drop }\end{array}$ & 0.14 & 0.42 \\
\hline 41 & $\begin{array}{l}\text { Helicteres } \\
\text { isora (L.) }\end{array}$ & Malvaceae & $\begin{array}{l}\text { Muradpha } \\
\text { lli }\end{array}$ & $\begin{array}{l}\text { Murad- } \\
\text { sheng }\end{array}$ & Herb & Fruit & $\begin{array}{l}\text { Diarrhoea, } \\
\text { Dysentry, }\end{array}$ & Paste & Oral & 0.14 & 0.28 \\
\hline 42 & $\begin{array}{l}\text { Ixora arborea } \\
\text { (Roxb.) }\end{array}$ & Rubiaceae & $\begin{array}{l}\text { Lokhandi } \\
\text { pala }\end{array}$ & $\begin{array}{l}\text { Kota- } \\
\text { gandhal }\end{array}$ & Tree & Leaves & $\begin{array}{l}\text { Diseases of } \\
\text { nervous } \\
\text { system , } \\
\text { weakness }\end{array}$ & Decoction & Oral & 0.28 & 0.42 \\
\hline 43 & $\begin{array}{l}\text { Kalanchoe } \\
\text { pinnata (Lam.) } \\
\text { Pers }\end{array}$ & Crassulaceae & Panphuti & $\begin{array}{l}\text { Parna- } \\
\text { phuti }\end{array}$ & Herb & Leaves & Diabetis & Juice & Oral & 0.28 & 0.28 \\
\hline
\end{tabular}




\begin{tabular}{|c|c|c|c|c|c|c|c|c|c|c|c|}
\hline 44 & $\begin{array}{l}\text { Jasminum } \\
\text { grandiflorum } \\
\text { L. }\end{array}$ & Oleaceae & $J a i$ & Jati & Climber & Leaves & $\begin{array}{l}\text { Mouth ulcer, } \\
\text { pyorrhoea }\end{array}$ & Decoction & Gargle & 0.14 & 0.28 \\
\hline 45 & $\begin{array}{l}\text { Lannea } \\
\text { coromandelica } \\
\text { (Houtt) Merr }\end{array}$ & Anacardiceae & $M o i$ & Jhingini & Tree & Bark & $\begin{array}{l}\text { Wound } \\
\text { healing }\end{array}$ & Decoction & Topical & 0.14 & 0.14 \\
\hline 46 & $\begin{array}{l}\text { Litsea } \\
\text { glutinosa } \\
\text { (Lour.)CB } \\
\text { Robins }\end{array}$ & Lauraceae & $\begin{array}{l}\text { Maidalak } \\
\text { di } \\
\text { Lenja }\end{array}$ & Medasak & Tree & $\begin{array}{l}\text { Bark, } \\
\text { Leaves }\end{array}$ & $\begin{array}{l}\text { Urinary } \\
\text { diseases, } \\
\text { tonic }\end{array}$ & $\begin{array}{l}\text { Decoction, } \\
\text { Powder }\end{array}$ & Oral & 0.28 & 0.71 \\
\hline 47 & $\begin{array}{l}\text { Melia } \\
\text { azedarach } \\
\text { (L.) }\end{array}$ & Meliaceae & Bakan & $\begin{array}{l}\text { Mahanim } \\
\text { ba }\end{array}$ & Tree & Bark & $\begin{array}{l}\text { Skin diseases, } \\
\text { Fever }\end{array}$ & Decoction & Oral & 0.14 & 0.28 \\
\hline 48 & $\begin{array}{l}\text { Mimosa } \\
\text { pudica(L.) }\end{array}$ & Leguminoceae & Lajvanti & Lajjalu & Creeper & Root & $\begin{array}{l}\text { Snake bite } \\
\text { Blood } \\
\text { purifier }\end{array}$ & Paste & Topical & 0.14 & 0.28 \\
\hline 49 & $\begin{array}{l}\text { Mucuna } \\
\text { pruriens(L.) } \\
\text { DC. }\end{array}$ & Leguminoceae & Khajkuhili & $\begin{array}{l}\text { Kapi- } \\
\text { kachhu }\end{array}$ & Climber & Seeds & $\begin{array}{l}\text { Scorpion bite, } \\
\text { Azzospermia }\end{array}$ & Powder & $\begin{array}{l}\text { Tropical, } \\
\text { oral }\end{array}$ & 0.14 & 0.28 \\
\hline 50 & $\begin{array}{l}\text { Ocimum } \\
\text { sanctum }(\mathrm{L} .)\end{array}$ & Labiateae & Tulas & Tulsi & Herb & Leaves & $\begin{array}{l}\text { Asthama, } \\
\text { Phelgm }\end{array}$ & Decoction & Oral & 0.14 & 0.28 \\
\hline 51 & $\begin{array}{l}\text { Randia } \\
\text { dumentorum } \\
\text { (Retz) Lam. }\end{array}$ & Rubiaceae & Fetal & $\begin{array}{l}\text { Madan- } \\
\text { phala }\end{array}$ & Tree & Root & Scorpion bite & Paste & Topical & 0.14 & 0.14 \\
\hline 52 & $\begin{array}{l}\text { Ricinus } \\
\text { communis (L.) }\end{array}$ & Euphorbiaceae & Erandi & Erand & shrub & Leaves & Jaundice & Juice & Oral & 0.14 & 0.14 \\
\hline 53 & $\begin{array}{l}\text { Plumbago } \\
\text { zeylanica }(\mathrm{L} .)\end{array}$ & Plunbaginaceae & Chitraka & Chitraka & Herb & Root & $\begin{array}{l}\text { Chronic skin } \\
\text { diseases, } \\
\text { Vitiligo }\end{array}$ & Paste & Topical & 0.14 & 0.28 \\
\hline 54 & $\begin{array}{l}\text { Pongamia } \\
\text { glabra (L.) }\end{array}$ & Leguminoceae & Karanji & Karanj & Tree & Leaves & $\begin{array}{l}\text { Diseases of } \\
\text { nervous } \\
\text { system }\end{array}$ & Decoction & Oral & 0.14 & 0.14 \\
\hline 55 & $\begin{array}{l}\text { Solanum } \\
\text { indicum } \\
\text { (Linn.) }\end{array}$ & Solanaceae & Bhui dorli & Brihati & Herb & Root & Dog bite & Paste & Topical & 0.14 & 0.14 \\
\hline 56 & $\begin{array}{l}\text { Scilla indica } \\
\text { (Roxb) }\end{array}$ & Liliaceae & Rankanda & $\begin{array}{l}\text { Van- } \\
\text { palandu }\end{array}$ & Herb & Tuber & $\begin{array}{l}\text { Cough, } \\
\text { Sotha, }\end{array}$ & Paste & Topical & 0.14 & 0.28 \\
\hline 57 & $\begin{array}{l}\text { Sterculia urens } \\
\text { (Roxb) }\end{array}$ & Malvaceae & Karucha & Karu & Tree & Gum & Bone fracture & Powder & Oral & 0.14 & 0.14 \\
\hline 58 & $\begin{array}{l}\text { Tephrosea } \\
\text { purpura } \\
\text { (L.)Pres }\end{array}$ & Fabaceae & Unhali & $\begin{array}{l}\text { Shar- } \\
\text { punkha }\end{array}$ & Herb & $\begin{array}{l}\text { Root, } \\
\text { Seed }\end{array}$ & $\begin{array}{l}\text { Snake bite, } \\
\text { colic } \\
\text { Spleenomegal } \\
\text { ae }\end{array}$ & Powder & $\begin{array}{l}\text { Oral, } \\
\text { Topical }\end{array}$ & 0.14 & 0.42 \\
\hline 59 & $\begin{array}{l}\text { Tinospora } \\
\text { cordifolia } \\
\text { (Willd.) }\end{array}$ & $\begin{array}{l}\text { Menisperm- } \\
\text { aceae }\end{array}$ & Gulvel & Guduchi & Climber & Stem & Arthritis, gout & Decoction & Oral & 0.42 & 0.71 \\
\hline 60 & $\begin{array}{l}\text { Tribulus } \\
\text { terristris (L.) }\end{array}$ & Zygophyllaceae & Sarata & Gokshur & Creeper & Fruit & $\begin{array}{l}\text { Urinary } \\
\text { diseases, } \\
\text { burning } \\
\text { micturation }\end{array}$ & $\begin{array}{l}\text { Decoction, } \\
\text { powder }\end{array}$ & Oral & 0.14 & 0.28 \\
\hline 61 & $\begin{array}{l}\text { Terminalia } \\
\text { arjuna Wight } \\
\text { \& Arn }\end{array}$ & Combretaceae & $\begin{array}{l}\text { Aajan } \\
\text { saal }\end{array}$ & Arjuna & Tree & Bark & $\begin{array}{l}\text { Heart } \\
\text { diseases, } \\
\text { Diabetis } \\
\text { Otalgia }\end{array}$ & $\begin{array}{l}\text { Decoction, } \\
\text { Leaves } \\
\text { juice }\end{array}$ & $\begin{array}{l}\text { Oral, } \\
\text { eardrop }\end{array}$ & 0.14 & 0.42 \\
\hline 62 & $\begin{array}{l}\text { Tridex } \\
\text { procumbens } \\
\text { (L.) }\end{array}$ & Asteraceae & $\begin{array}{l}\text { Ekdandi, } \\
\text { Ghavpala, } \\
\text { dagdi } \\
\text { pala }\end{array}$ & $\begin{array}{l}\text { Kamber- } \\
\text { modi }\end{array}$ & Herb & Leaves & Cuts, wounds & Juice & Topical & 0.28 & 0.85 \\
\hline 63 & $\begin{array}{l}\text { Ventilago } \\
\text { denticulata } \\
\text { (Willd.) }\end{array}$ & Rhamnaceae & Raktapitti & Raktavalli & Climber & Root & Menorrhagia & Decoction & Oral & 0.14 & 0.14 \\
\hline 64 & $\begin{array}{l}\text { Vitex negundo } \\
\text { (L.) }\end{array}$ & Laminaceae & Nirgudi & Nirgundi & Shrub & Leaves & $\begin{array}{l}\text { Vataroga, } \\
\text { Shotha }\end{array}$ & Decoction & Oral & 0.28 & 0.28 \\
\hline 65 & $\begin{array}{l}\text { Cardiospermu } \\
\text { m helicacabum } \\
\text { (L.) }\end{array}$ & Sapindaceae & $\begin{array}{l}\text { Kapal- } \\
\text { phodi }\end{array}$ & Indravalli & Climber & Leaves & $\begin{array}{l}\text { Rhumatism, } \\
\text { arthritis }\end{array}$ & Poultice & Topical & 0.14 & 0.28 \\
\hline
\end{tabular}

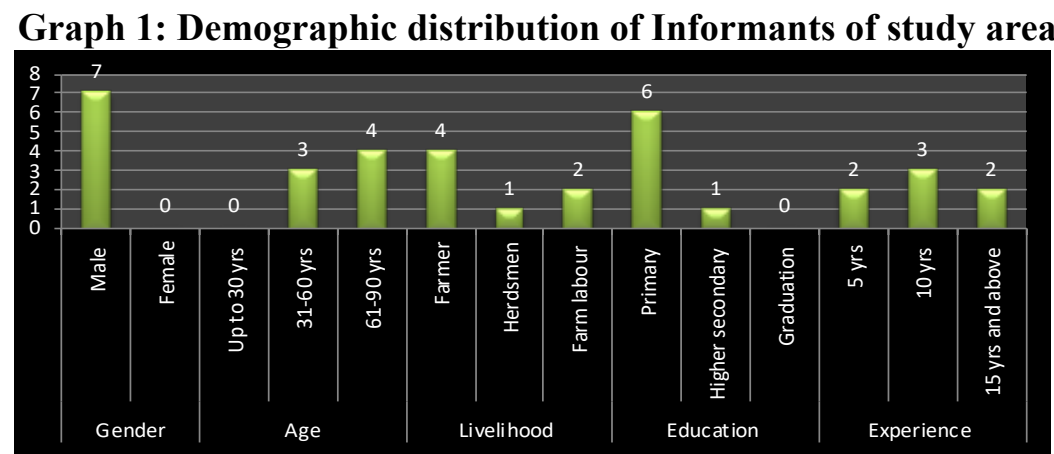


Bharat Rathi et.al., Ethno-botanical Survey on Medicinal plants used by Tribes of Karanja (Ghadge) Tahsil

Graph 2 : Habitat of the study area

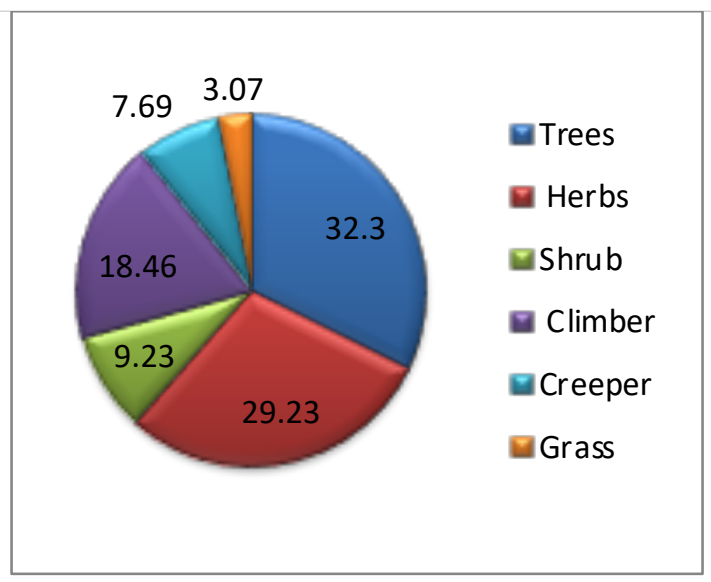

Graph 3 : Percentage of Plants parts used for the preparation of medicine

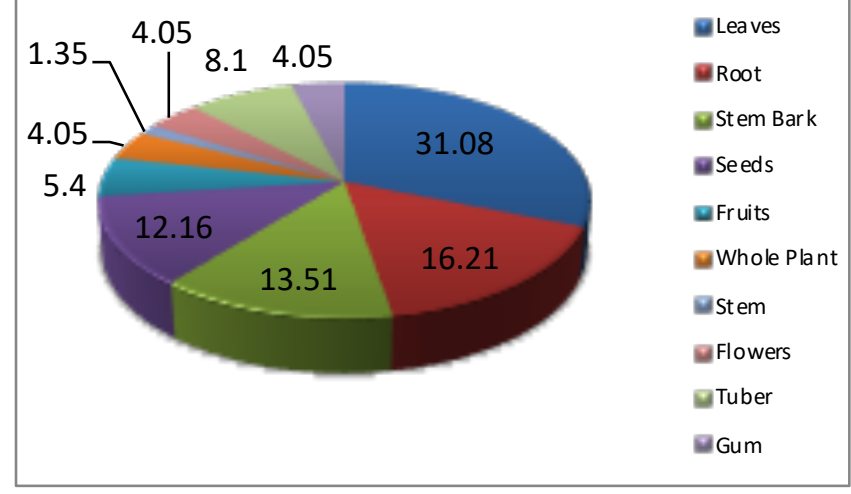

Graph 4 : Proportional contribution of herbal preparations in the treatment

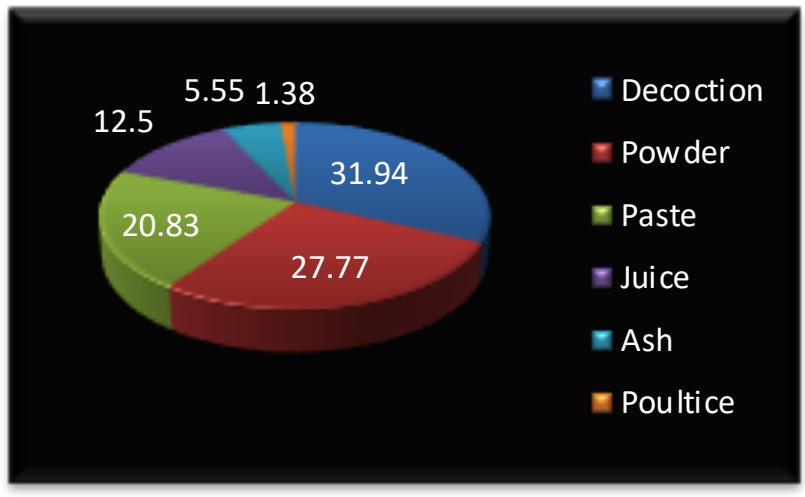

Graph 5 : Routes of administration of prepared medicine in the study area

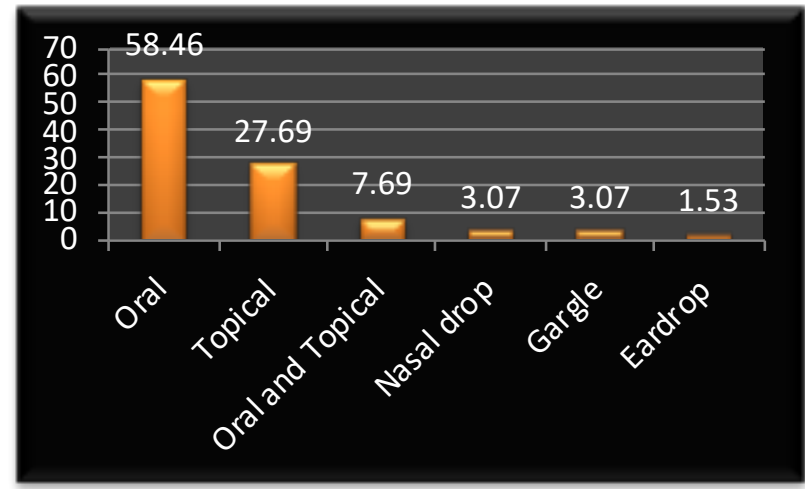

Table 2: Commonly used herbal drugs of the study area along with FL, RPL and ROP value

\section{S.N. Name of drug}

Adhatoda vasica Nees

Tinospora cordifolia Willd.

Achyranthes aspera Linn.

Ailanthus excels Roxb.

Arundo donax Linn.

Bacopa monnieri Linn.

Butea monosperma Lam.

Euforbia nerifolia Linn.

Citrullus colocynthis(L.) Schrad

Kalonchoe pinnata (Lam.) Pers

Ixora arborea Roxb.

Litsea glutinosa (Lour.)CB Robins

Vitex negundo Linn.

Tridex procumbens Linn.

\begin{tabular}{|l|}
$\mathbf{N}$ \\
4 \\
3 \\
2 \\
2 \\
2 \\
2 \\
\hline 2 \\
2 \\
2 \\
\hline 2 \\
2 \\
2 \\
2 \\
\hline 2 \\
\hline
\end{tabular}

\begin{tabular}{|l|l|}
\hline $\mathbf{N}$ & $\mathbf{N}$ \\
\hline 4 & 4 \\
\hline 3 & 4 \\
\hline 2 & 3 \\
\hline 2 & 3 \\
\hline 2 & 2 \\
\hline 2 & 5 \\
\hline 2 & 2 \\
\hline 2 & 2 \\
\hline 2 & 3 \\
\hline 2 & 2 \\
\hline 2 & 2 \\
\hline 2 & 3 \\
\hline 2 & 2 \\
\hline 2 & 3 \\
\hline
\end{tabular}

\begin{tabular}{|l|l|l|l|l|}
\hline Disease treated & $\mathbf{N}$ & $\mathbf{R} L^{*}$ & $\mathbf{F L}^{* *}$ & $\mathbf{R O P * * *}$ \\
\hline Cough \& Bronchitis & $\mathbf{p}$ & 0.28 & 100 & 28 \\
\hline Arthritis & 3 & 0.42 & 100 & 42 \\
\hline Scorpion bite & 2 & 0.42 & 100 & 42 \\
\hline Tonic & 2 & 0.42 & 100 & 42 \\
\hline Himorrhoides & 2 & 0.28 & 100 & 28 \\
\hline Nervous disorder & 1 & 0.71 & 50 & 35 \\
\hline Diabetes & 1 & 0.28 & 50 & 14 \\
\hline Eczema & 2 & 0.14 & 100 & 14 \\
\hline Worm infestation & 2 & 0.42 & 100 & 42 \\
\hline Renal calculi & 1 & 0.28 & 50 & 14 \\
\hline Arthritis & 2 & 0.14 & 100 & 14 \\
\hline Urinary disease & 2 & 0.28 & 100 & 28 \\
\hline Diseases of nervous system & 2 & 0.28 & 100 & 28 \\
\hline Cuts \& wounds & 2 & 0.28 & 100 & 28 \\
\hline
\end{tabular}

$\mathrm{N}$ - Number of total informants citing the species for any given disease ND - Number of diseases treated

$\mathrm{Np}$ - No. of informants who asserted a use of certain herb for a specific ailment

FL - Fidelity level, RPL- Relative popularity level, ROP- Rank order priority

Table 3: Showing the ICF value of used herbal plants against various disease categories

\begin{tabular}{|l|l|c|c|r} 
No. & Disease categories & $\begin{array}{l}\text { Number of use } \\
\text { reports }\end{array}$ & No. of plants used & ICF* Value \\
\hline 1 & Gastro-Intestinal diseases & 17 & 15 & 0.12 \\
\hline 2 & Dermatological Diseases & 22 & 18 & 0.19 \\
\hline 3 & Respiratory Diseases & 15 & 10 & 0.35 \\
4 & Genito-Urinary Diseases & 22 & 19 & 0.14 \\
\hline 5 & Skeleto-Muscular Disorders & 17 & 13 & 0.18 \\
6 & Poisonous Bites & 15 & 13 & 0.14 \\
\hline 7 & Cardio-vascular diseases & 1 & 1 & 0.00
\end{tabular}


International Journal of Ayurvedic Medicine, Vol 12 (1), 43-52

\begin{tabular}{|l|l|l|l|l|}
\hline 8 & Endocrinal Disorders & 4 & 4 \\
\hline 9 & Ear, Nose, Throat (ENT) \& mouth related problems & 5 & 0.00 \\
\hline 10 & Reticulo endothelial system & 2 & 5 \\
\hline 11 & Nervous disorders & 6 & 2 \\
\hline 12 & General Health & 9 & 0.00 \\
\hline
\end{tabular}

\section{Discussion}

Demographic/Personal Information on Respondents:

Total 7 tribal traditional healers commonly known as Vaidu were interviewed during the survey period. All were men ranges in the age of 48 to 82 years having the practicing experience of 5 to 25 years. All these tribes speak Marathi language for communication, belonged to farmer, herdsmen and farming labor category and hardly have had primary education. All were primarily depending on an agricultural and forest resource for their survival(Gragh1).

\section{Diversity of Medicinal plant}

Present investigation revealed information on 69 plant species but after cross checking in follow up visits 65 plant species related to 46 families were verified, authenticated and then documented (Table 1) as being used to various ailments. The most representative family was Leguminoceae with 6 species, Acanthaceae 3 species, Asclepiadaceous, Cucurbitaceae, Convovulaceae, Combritaceae, Euphorbiceae, Gramineae, Fabaceae, Laminaceae, Maliaceae, Malvaceae, Rubiaceae and Zygophyllaceae with two species each and the remaining families were represented by one species each associated with the treatment of the reports. Informants generally share the name of plants in local language used in the treatment of various diseases. This was inconsonance with Singh (2008) who reported that plants are generally known by their colloquial names throughout the world (15). The colloquial names help in understanding the ethno botanical study of a particular tribe or region. Due to the lack of uniformity and consistency local names are not recommended directly for scientific accounts, yet they may certainly play a vital role in search of new useful plants or new indications of known plants (16).

\section{Habitat}

The habitat of the documented plant species in the study area is mentioned in graph 2. Total 6 types of habitat were identified out of the 65 plant species discussed/reported. They were trees (20 species), herbs (19 species), Shrubs (07 species), climbers (12 species), creepers (05 species) and grass (02 species). This classification revealed that trees $(32.30 \%)$ and herbs $(29.23 \%)$ were the routinely used plant habits by the traditional healers for treating the various disease conditions in the study area. This could be associated to the high availability throughout the year and usefulness of trees and herbs in the study area, making them available to the users easily (17).

\section{Plant parts used}

Though all plant parts are important as a medicine, however it is observed (graph 3) that traditional healers utilized leaves as the most common plant part with $31.08 \%$ applications in medicinal preparations, followed by roots $(16.21 \%)$, stem bark (13.51\%) Seeds $(12.16 \%)$, fruits $(5.40 \%)$ tuber $(8.10 \%)$, flowers, whole plant, Gum and resin (each $4.05 \%$ respectively) and stem (1.35\%). These findings are congruent with the study conducted by Shosan L. et al (18), Senthilkumar K et al (19) and Chandrakumar Patale et al (20). It is obvious that the use of leaves is beneficial for the survival of medicinal plants as compared to the collection of stem, root and whole plant which may cause severe menace to local flora and fauna.

Traditional healers of the study area have had the sound knowledge with respect to medicinal preparations for the treatments of various ailments. Most of the preparations used were in the form of decoction, juice, powder, paste, ash and poultice. Decoction was the choice of preparation (23 applications), followed by powder, paste and juice (20, 15 and 09 applications respectively) whereas ash (black burned powder) and poultice were used in 04 and 01 applications respectively (Graph 4). It aids the traditional healers to utilize the herbal drugs in various forms and techniques to treat the diseases and to compete with easy dispensing and palatability (21). Findings of broad range of medicine preparation methods have also been reported in previous studies (22-23).The broad use of decoction and powder found in the survey is congruent with the ethnomedicinal survey conducted in various tribes of India and other countries (24-26). Internal uses of medicines (58.46\%) were predominant over external or topical uses $(27.69 \%)$, both oral and topical $(7.69 \%)$, nasal drop and gargle $(3.07 \%$ each) and only $1.53 \%$ administered as eardrop (Graph 5).

\section{Informant Consensus factor}

Informant Consensus factor was calculated by classifying the reported diseases into 12 types of disease categories based on their use reports (Table 3). It is observed that dermatological and genitourinary diseases were dominated with each 22 use reports followed by gastrointestinal diseases and skeleto muscular disorders (17 use reports each respectively) and respiratory diseases and poisonous bites (15 use reports each respectively).Thus findings from the study revealed that dermatological diseases and genitourinary disorders are common in the study area. Personal unhygienic conditions prevalent in the study area may be reason to develop these conditions. Similar findings are congruent with the study conducted by Muhammad Umair et al Punjab Pakistan (27). The ICF values of different diseases categories were ranged from ' 0 '(cardiovascular diseases, endocrinal diseases, ENT disorders, Reticulo endothelial system \& general health) to 0.35 for respiratory disorders. Thus for all disease categories the 
average ICF value was 0.12 . Higher ICF value (0.35) calculated for respiratory disorders which indicate that genuine species of herbs are utilized by tribal healers because of their authenticity with respect to disease curing capacity. It also tests the consistency of informant's knowledge regarding plants species for treating a particular illness category. However low ICF indicates that informants have no resemblance in using the herbal drugs while treating the diseases.

\section{Relative frequency of citation (RFC)}

RFC signifies the local importance of each species in study area. RFC of reported species ranged in between 0.14 To 0.57 . The higher RFC was observed for Adhatoda vasica (0.57) followed by Tinospora cordifolia (0.42) and Achyranthus aspera, Ailanthus excelsa, Arundo donax, Bacopa monnieri, Citrulus colosynthis, Euphorbia nerifolia, Ixora arborea, Kalanchoe pinnata, Litsea glutinosa, Tridax procumbens and Vitex nigundo (0.28) respectively. It means that these species were well known to the informants of the study area. (Table 1) Those medicinal plant species having high RFC must be further assessed for phytochemical analysis and pharmaceutical analysis to identify their active constituents.

\section{Use value (UV)}

Data collected from the present study it is observed that UV ranges from 0.14 to 0.85 . The highest UV calculated for Adhatoda vasica, Tridex procumbens, Ailanthus excelsa (UV $=0.85$ each) followed by and Tinosphora cardifolia, Bacopa monnieri, Citrulus colocynthis \& Litsea glutinosa (UV= 0.71 each). This indicated the sound knowledge of informants regarding various plant species in treating the various ailments. Recent advance researches on few herbal drugs like Azadirachta indica, Tinospora cordifolia, Bacopa monnieri, Ocimum sanctum have revealed the significant immune boosting and antiviral activity also (28-29).

Lowest UV of Balanites aegyptica, Kalanchoe pinnata, Solanum indicum \& Ventilago denticulate (UV $=0.14$ each ) may be due to the low accessibility of these species in the study area. Plant species with high UV should be further studied for ethno-pharmacological screening to find out the active compounds.

\section{Related popularity level (RPL)}

The highest RPL value calculated for Bacopa monnieri (0.71) followed by Tinospora cordifolia, Achyranthes aspera, Citrulus colocynthis (0.42). It means not a single drug have achieved complete popularity level. May be due to each drug is considered as best in specific condition.

\section{Fidelity level (FL)}

The fidelity level (FL) of the 14 most important plant species ranged from 50 to $100 \%$. The high FL indicates the frequency and maximum use of the plant species for treating a particular ailment by the informants. Adhatoda vasica, Tinospora cordifolia, Achyranthes aspera, Ailanthus excelsa, Arundo donax,
Euphorbia nerifolia, Citrulus colocynthis, Ixora arborea, Litsea glutinosa, Vitex negundo, Tridax procumbens showed $100 \%$ FL against the diseases shown in table 2.

Study conducted by Srithi K et al (2009) and Bibi $\mathrm{T}$ et al (2014), Vitex negundo and Ixora arborea depicted $100 \%$ FL against diseases of nervous system respectively (30-31). In another study, Ranunculus sceleratus, Moringa oleifera, Tribulus terrestris were claimed as having antiurolithiatic property to treat urinary disorder (32-33).

\section{Rank order priority (ROP)}

ROP is a correction factor, used for appropriate ranking of the plant species with different FL and RPL values. Out of the highly ranked 14 species, only four species attained ROP near 50. Based on ROP value Achyranthus aspera, Ailanthus excelsa, Citrulus colocynthis and Tinospora cordifolia were widely utilized species with ROP $=42$. The other plant species with significant ROP was Bacopa monnieri ( $\mathrm{ROP}=35$ )

From the above findings it is recommended that the plant species used by the traditional healers in specific disease condition and species with high RPL value could be studied further to screen bioactive compounds and their pharmacological activities to introduce novel drugs.

\section{Conclusion}

Present study concluded that the knowledge and applications of traditional herbal medicine for the treatment of various diseases prevalent among the tribes located in Karanja Ghadge Tahsil of Wardha district is still a major part of their life and culture. Tribal communities have a strong belief in the efficacy and success of these species grow around them and the results of the study provided evidences that the plant species continued to play vital role in the healthcare system of this community. Lacking the interest among the younger generations of tribal people in traditional medicine is a matter of concern. The present work would be useful in identification of newer species and their therapeutic applications. Ethno-botanical data of plant species may be helpful to policy makers which are under threat due to over exploitation. Traditional medicinal preparations may pave a way to develop advance and modified drug dosage forms so that can be administered by different routes over traditional routes effectively.

\section{Acknowledgements}

Author would like to thank DMIMSU for motivating and providing all necessary help for writing this article.

\section{Funding Support \\ DMIMS (Deemed to be University) Sawangi (Meghe) Wardha.}

\section{Conflict of Interest: None}




\section{References}

1. Rath C, Bonthu S, Tripathi A, Chincholikar M, Mangal A. Medico- Ethno-botanical Survey: An Overview of CCRAS Contributions, Journal of Drug Research in Ayurvedic Sciences, JulySeptember (2017; 2(3);188-240

2. Rathi B, Rathi R. Principals of ethical Ayurveda prescription writing in clinical practice: A literature review. J Datta Meghe Inst Med Sci Univ 2019;14: S97-102.

3. Acharya Charak, Charak Samhita, Gaud B.(editor) Ayurved Deepika Vyakhya, 1 1st edn, Sutra Sthana 1/120, New Delhi, Rashtriya Ayurveda Vyaspeeth, 2011, 107p

4. Acharya Susruta, Sushruta Samhita. Sharma PV (editor), Reprint Vol I, Sutra Sthana, Bhumipravibhaga vijnaaneey Adhyaya, 36/10. Varanasi, Chaukhambha Surbharati; 2010, 345p

5. Pie S.J. Ethno-medicinal approaches of traditional medicine studies: some experiences form Asia. Pharmaceuticals Biology, 2001; 39: Supl 1;74-79.

6. Posey D. Traditional Knowledge, Conservation and the Rain Forest Harvest. In: Sustainable Harvest and Marketing of Rain Forest Products. Island Press First edition, Washington DC), 1992; 46- 50p.

7. Perumalsamy RP, Ignacimuthu S. Antibacterial activity of some of folklore medicinal plants used by tribal's in Western Ghats of India. $J$ of Ethnopharmacology. 2000; 69(1); 63-71

8. Rathi B, Rathi R. Ethno Medicinal Documentation of Plants used in the Treatment of Skin Diseases By the Tribal's of Karanja Ghadge Tahsil of Wardha District. Journal of School of Advanced Studies, 2019;2(1);22-6.

9. Trotter RT, Logan MH. Informant consensus: a new approach for identifying potentially effective medicinal plants. In: Etkin NLE, editor. Plants in Indigenous Medicine and Diet, Biobehavioural Approaches. Bedford Hills, NY: Redgrave Publishers, 1986, 91-112p

10. Phillips O, Gentry AH. The useful plants of Tambopata, Peru: Statistical hypotheses tests with a new quantitative technique. Economic Botany. 1993; 47(1) ;15-32

11. Ilker U, Suleyman B, Nurettin Y \& Yunus D. The investigation and quantitative ethnobotanical evaluation of medicinal plants used around Izmir province, Turkey. Journal of Medicinal Plants Research, 2009; 3(5) ;345-367

12. Alexiades MN \& Sheldon JW. Selected guidelines for ethnobotanical research: A Field Manual. The New York Botanical Garden, 1996; 53-94p

13. Kadir MF, Bin Sayeed MS \& Mia MM. Ethnopharmacological survey of medicinal plants used by indigenous and tribal people in Rangamati, Bangladesh. Journal of Ethnopharmacology. 2012; 144(3); 627- 637

14. Arshad M, Ahmad M, Ahmed E, Saboor A, Abbas A \& Sadiq S. An ethnobiological study in Kala Chitta hills of Pothwar region, Pakistan: multinomial logit specification. Journal of Ethnobiology and Ethnomedicine. 2014; 10(1);10: 13

15. Singh, H. Importance of Local Names of Some Useful Plants in Ethnobotanical Study. Indian Journal of Traditional Knowledge, 2008; 7(2) ; 365-370

16. Erinoso, S.M. and Aworinde, D.O. Ethnobotanical Survey of Some Medicinal Plants Used in Traditional HealthCare in Abeokuta Areas of Ogun State, Nigeria. African Journal of Pharmacy and Pharmacology, 2012 ; 6(18) ;1352-1362

17. Rathi B, Rathi R. Quantitative analysis of Medicinal plants used by the traditional healers of Karanja block of Wardha district for treating musculoskeletal disorders. International Journal of Ayurvedic Medicine. 2020 ;11(2) ;175-83

18. Shosan, L.O., Fawibe, O.O., Ajiboye, A.A., Abeegunrin, T.A. and Agboola, D.A. Ethnobotanical Survey of Medicinal Plants Used in Curing Some Diseases in Infants in Abeokuta South Local Government Area of Ogun State, Nigeria. American Journal of Plant Sciences, 2014; 5(21); 3258-3268.

19. Senthilkumar K., Aravindhan V., Rajendran A, Ethnobotanical Survey of Medicinal Plants Used by Malayali Tribes in Yercaud Hills of Eastern Ghats, India, Journal of Natural Remedies , 2013;13(2); 129-132.

20. Chandrakumar Patale, Praveenkumar Nasare, Sushama Narkhede, Ethnobotanical studies on the medicinal plants of Darekasa Hill range of Gondia district, Maharashtra, India. Int $J$ of Res in Plant Sci. 2015; 5(1); 10-16.

21. Rathi B, Rathi R, Rajput DS. Pharmaceutical standardization of Avalgujadi Lepaguti. J. Indian Sys Med. 2016;4(2);72-76

22. Ramya S, Alaguchamy N, Maruthappan VM, Sivaperumal $\mathrm{R}$ \& Sivalingam $\mathrm{M}$ et al. Wound Healing Ethnomedicinal Plants Popular among Malayali Tribes in Vattal Hills Dharmapuri TN India. Ethnobotanical Leaflets , 2009;13: 1257-1271.

23. Song MJ, Kim H, Heldenbrand B, Jeon J, Lee S. Ethnopharmacological survey of medicinal plants in Jeju Island, Korea. Journal of ethnobiology and ethnomedicine. 2013 Dec;9(1):1-8.

24. Bankar MV, Bhosle NP, Ethnobotanical Survey Of Medicinal Plants In Ajanta Region (MS) India, Journal of Pharmacy and Biological Sciences. 2017;12(6) (Ver. II);59-64.

25. Jenny MO, Suganthi A, Ethnobotanical survey on medicinal plants used by tribal people in Attappady, Kerala, International Journal of Pharmaceutical Science and Research, January 2017; 2(1);17-23.

26. Mahmood A, Mahmood A, Malik RN, Shinwari ZK. Indigenous knowledge of medicinal plants from Gujranwala district, Pakistan. Journal of Ethnopharmacology.2013;148(2);714-723.

27. Umair M, Altaf M, Abbasi AM. An ethnobotanical survey of indigenous medicinal plants in Hafizabad district, Punjab-Pakistan. PloS one. 2017 Jun 2;12(6); e0177912. 
28. Rathi R, Rathi B. COVID 19 Pandemic and Preventive Footsteps. Int $\mathrm{J}$ Ayu Pharm Chem. 2020;12(3) ;100-6

29. Bhokardankar P, Rathi B, Khan M, Rathi R. COVID-19 pandemic: Home remedies for immunity boosting. International Journal of Research in Pharmaceutical Sciences. 2020 Mar 11;11(Special Issue 1). 734-738.

30. Srithi K, Balslev H, Wang pakapattanawong $\mathrm{P}$, Srisanga P, Trisonthi C. Medicinal plant knowledge and its erosion among the Mien (Yao) in northern Thailand. Journal of Ethnopharmacology. 2009; $123(2) ; 335-342$
31. Bibi T, Ahmad M, Tareen RB, Tareen NM, Jabeen R, Rehman SU, Sultana S, Zafar M, Yaseen G. Ethnobotany of medicinal plants in district Mastung of Balochistan province-Pakistan. Journal of ethnopharmacology. 2014 Nov $18 ; 157 ; 79-89$.

32. Mikawlrawng K and Kumar S. Current scenario of urolithiasis and the use of medicinal plants as antiurolithiatic agents in Manipur (North East India): a review. International Journal of Herbal Medicine. 2014; 2(1) ;1 -12.

33. Bhokardankar PS, Rathi B. Indigenous wisdom of Ayurvedic drugs to treat Urinary tract infections. International Journal of Ayurvedic Medicine. 2020;11(3) ;370-7. 